\title{
Retrial queues and related models
}

\author{
Antonio Gómez-Corral ${ }^{1}$. Tuan Phung-Duc ${ }^{2}$
}

Published online: 22 August 2016

C) Springer Science+Business Media New York 2016

This issue of the Annals of Operations Research is devoted to recent research that significantly enhances our ability to analyze retrial queues and related models, either mathematically or algorithmically. The main source of papers for this special issue has been the 10th International Workshop on Retrial Queues (WRQ'14), held in Tokyo from the 24th to the 26th of July 2014, but also some manuscripts of researchers who did not attend the workshop were considered for publication. The workshop was truly an international event which gathered researchers from ten countries all over the world, and it continued a tradition of scientific meetings on stochastic modelling in retrial queues and their applications in daily life systems, call centers, telecommunication systems, and computer networks. Special thanks go to all the people who attended the WRQ' 14 meeting and who were the main contributors to its success.

The special issue consists of fourteen contributions - two of which are invited survey papers written by specialists in retrial queues and related models: "A survey of retrial queueing systems," by Jeongsim Kim and Bara Kim, and "Retrial queueing models in discrete time: a short survey of some late arrival models," by Rein Nobel, which show analytical results ranging from the stability condition of the underlying stochastic process to a variety of performance measures, including the queue length, waiting times, busy period and tail asymptotics of the queue length distribution, and the waiting time distribution, among others. In the paper by Jeongsim Kim and Bara Kim, the interest is mainly in retrial queues in continuous-time; more particularly, the focus is first on the well-known $M / G / 1$ retrial queue and then on various variants where the Poisson arrival stream is replaced by either a compound Poisson process or a batch Markovian arrival process. The multiserver case is also studied in terms of the standard $M / M / c$ retrial queue and the $M / M / c$ retrial queue

\footnotetext{
Tuan Phung-Duc

tuan@sk.tsukuba.ac.jp

Antonio Gómez-Corral antonio.gomez@icmat.es

1 Instituto de Ciencias Matemáticas CSIC-UAM-UC3M-UCM, Madrid, Spain

2 University of Tsukuba, Tsukuba, Japan
} 
with nonpersistent customers. Rein Nobel presents an interesting overview on single-server retrial queues in discrete-time, thus focusing on retrial queueing models that have received less attention in the literature than the more traditional continuous-time analogues. The starting point for Rein Nobel is the discrete-time single-server retrial queue with the late arrival setup and delayed access (LARS-DA model), for which a precise description is first presented and then modified appropriately to incorporate various queueing features, such as abandonments, feedback, priorities, and a tolerant server, into the model description. It is a pleasure for us to acknowledge the excellent work made by Jeongsim Kim, Bara Kim and Rein Nobel.

According to their theoretical and application-oriented contents and methodological and/or practical interest, the remaining papers in the present issue can be classified into four groups, as follows.

Asymptotics and structured Markov chains

Finite sources

Queueing features, including balk arrivals, impatience, priorities, and reliability

Related models and applications

We wish to thank all the authors who submitted their papers to this special issue and we express our deepest gratitude to more than fifty specialists who acted as reviewers of these papers. To their meticulousness and high standards, we (guest editors), the authors, and you (the reader) owe many thanks. Last but not least we sincerely thank the Editor-in-Chief, Professor Endre Boros, and the Publications Manager, Katie D’Agosta, for their support and assistance. 